\title{
Two-channel sampling in wavelet subspaces
}

\author{
J.M. Kim and K.H. Kwon
}

\begin{abstract}
We develop two-channel sampling theory in the wavelet subspace $V_{1}$ from the multi resolution analysis $\left\{V_{j}\right\}_{j \in \mathbb{Z}}$. Extending earlier results by G. G. Walter [11], W. Chen and S. Itoh [2] and Y. M. Hong et al [5] on the sampling theory in the wavelet or shift invariant spaces, we find a necessary and sufficient condition for two-channel sampling expansion formula to hold in $V_{1}$.
\end{abstract}

\section{Indroduction}

The classical Whittaker-Shannon-Kotel'nikov(WSK) sampling theorem [4] states that any signal $f(t)$ with finite energy and the bandwidth $\pi$ can be completely reconstructed from its discrete values by the formula

$$
f(t)=\sum_{n=-\infty}^{\infty} f(n) \frac{\sin \pi(t-n)}{\pi(t-n)} .
$$

WSK sampling theorem has been extended in many directions (see [1], [2], [5], [6], [7], [8], [10], [11], [12] and references therein). G. G. Walter [11] developed a sampling theorem in wavelet subspaces, noting that the sampling function sinct $:=\sin \pi t / \pi t$ in the WSK sampling theorem is a scaling function of a multi-resolution analysis. A. J. E. M. Janssen [6] used the Zak transform to generalize Walter's work to regular shifted sampling. Later, W. Chen and S. Itoh [2] (see also [12]) extended Walter's result further by relaxing conditions

Key Words: two-channel sampling, wavelet subspaces, Riesz basis

2010 Mathematics Subject Classification: Primary 42C15

Received: 26 April, 2014.

Revised: 30 May, 2014.

Accepted: 27 June, 2014. 
on the scaling function $\phi(t)$. Recently in [5],[8] general sampling expansion are handled on shift invariant spaces([9]). In this work, we find a necessary and sufficient condition for two-channel sampling expansion to hold in the wavelet subspace $V_{1}$ of a multi resolution analysis $\left\{V_{j}\right\}_{j \in \mathbb{Z}}$.

\section{Preliminaries}

For a measurable function $f(t)$ on $\mathbb{R}$, we let

$$
\|f(t)\|_{0}:=\sup _{|E|=0} \inf _{\mathbb{R} \backslash E}|f(t)| \quad \text { and } \quad\|f(t)\|_{\infty}:=\inf _{|E|=0} \sup _{\mathbb{R} \backslash E}|f(t)|
$$

be the essential infimum and the essential supremum of $|f(t)|$ on $\mathbb{R}$ respectively, where $|E|$ is the Lebesgue measure of $E$. For any $f(t) \in L^{2}(\mathbb{R}) \cap L^{1}(\mathbb{R})$, we let

$$
\mathcal{F}(f)(\xi)=\hat{f}(\xi):=\int_{-\infty}^{\infty} f(t) e^{-i t \xi} d t
$$

be the Fourier transform of $f(t)$ so that $\frac{1}{\sqrt{2 \pi}} \mathcal{F}(\cdot)$ becomes a unitary operator on $L^{2}(\mathbb{R})$. A sequence $\left\{\phi_{n}: n \in \mathbb{Z}\right\}$ in a Hilbert space $H$ is called a Riesz sequence if $\left\{\phi_{n}: n \in \mathbb{Z}\right\}$ is a Riesz basis of the closed subspace $V:=\overline{\operatorname{span}}\left\{\phi_{n}: n \in \mathbb{Z}\right\}$ of $H$.

Definition 1. A function $\phi(t) \in L^{2}(\mathbb{R})$ is called a scaling function of a multiresolution analysis (MRA in short) $\left\{V_{j}\right\}_{j \in \mathbb{Z}}$ if the closed subspaces $V_{j}$ of $L^{2}(\mathbb{R})$,

$$
V_{j}:=\overline{\operatorname{span}}\left\{\phi\left(2^{j} t-k\right): k \in \mathbb{Z}\right\}, \quad j \in \mathbb{Z}
$$

satisfy the following properties;

1. $\cdots \subset V_{-1} \subset V_{0} \subset V_{1} \cdots$;

2. $\bar{U} V_{j}=L^{2}(\mathbb{R})$;

3. $\cap V_{j}=\{0\}$;

4. $f(t) \in V_{j}$ if and only if $f(2 t) \in V_{j+1}$;

5. $\{\phi(t-n): n \in \mathbb{Z}\}$ is a Riesz basis of $V_{0}$.

The wavelet subspace $W_{j}$ is the orthogonal complement of $V_{j}$ in $V_{j+1}$ so that $V_{j+1}=V_{j} \oplus W_{j}$. Then there is a wavelet $\psi(t) \in L^{2}(\mathbb{R})$ that induces a Riesz basis $\left\{\psi\left(2^{j} t-k\right): k \in \mathbb{Z}\right\}$ of $W_{j}$. Moreover, $\left\{\phi\left(2^{j} t-k\right), \psi\left(2^{j} t-k\right): k \in \mathbb{Z}\right\}$ forms a Riesz basis of $V_{j+1}$. 
For any $\phi(t) \in L^{2}(\mathbb{R}),\{\phi(t-n): n \in \mathbb{Z}\}$ is a Bessel sequence if there is a constant $B>0$ such that

$$
\sum_{n \in \mathbb{Z}}|\langle f(t), \phi(t-n)\rangle|^{2} \leq B\|f\|_{L^{2}(\mathbb{R})}^{2}, \quad f \in L^{2}(\mathbb{R})
$$

or equivalently (see Theorem 7.2 .3 in [3]) $G_{\phi}(\xi):=\sum_{n \in \mathbb{Z}}|\hat{\phi}(\xi+2 n \pi)|^{2} \leq B$ a.e. on $[0,2 \pi]$.

Lemma 1. (Lemma 2.2 in [5] and Lemma 7.2.1 in [3]) Let $\phi(t) \in L^{2}(\mathbb{R})$ be such that $\{\phi(t-k): k \in \mathbb{Z}\}$ is a Bessel sequence. Then, for any $\left\{c_{k}\right\}_{k \in \mathbb{Z}} \in$ $l^{2}, \sum_{k \in \mathbb{Z}} c_{k} \phi(t-k)$ converges in $L^{2}(\mathbb{R})$ and

$$
\mathcal{F}\left(\sum_{k \in \mathbb{Z}} c_{k} \phi(t-k)\right)=\left(\sum_{k \in \mathbb{Z}} c_{k} e^{-i k \xi}\right) \hat{\phi}(\xi) .
$$

For any $\mathbf{c}=\left\{c_{k}\right\}_{k \in \mathbb{Z}} \in l^{2}$, let $\widehat{\mathbf{c}}(\xi):=\sum_{k \in \mathbb{Z}} c_{k} e^{-i k \xi}$. Then, $\widehat{\mathbf{c}}(\xi) \in L^{2}[0,2 \pi]$ or $C[0,2 \pi]$ if $\left\{c_{k}\right\}_{k \in \mathbb{Z}} \in l^{2}$ or $l^{1}$ respectively.

Lemma 2. If $\mathbf{a}=\left\{a_{k}\right\}_{k \in \mathbb{Z}}, \mathbf{b}=\left\{b_{k}\right\}_{k \in \mathbb{Z}} \in l^{2}$ and $\widehat{\mathbf{a}}(\xi) \in L^{\infty}[0,2 \pi]$, then $\mathbf{a} * \mathbf{b}:=\left\{\sum_{j \in \mathbb{Z}} a_{j} b_{k-j}\right\}_{k \in \mathbb{Z}} \in l^{2}$ and

$$
\widehat{\mathbf{a} * \mathbf{b}}(\xi)=\widehat{\mathbf{a}}(\xi) \widehat{\mathbf{b}}(\xi) .
$$

Proof. Since $\widehat{\mathbf{a}}(\xi) \in L^{\infty}[0,2 \pi]$ and $\widehat{\mathbf{b}}(\xi) \in L^{2}[0,2 \pi], \widehat{\mathbf{a}}(\xi) \widehat{\mathbf{b}}(\xi) \in L^{2}[0,2 \pi]$. Hence we can expand $\widehat{\mathbf{a}}(\xi) \widehat{\mathbf{b}}(\xi)$ into its Fourier series $\sum_{n} c_{n} e^{-i n \xi}$ in $L^{2}[0,2 \pi]$, where

$$
\begin{aligned}
c_{n} & =\frac{1}{2 \pi}\left\langle\widehat{\mathbf{a}}(\xi) \widehat{\mathbf{b}}(\xi), e^{-i n \xi}\right\rangle_{L^{2}[0,2 \pi]}=\frac{1}{2 \pi}\left\langle\sum_{k \in \mathbb{Z}} a_{k} e^{-i k \xi},\left(\sum_{k \in \mathbb{Z}} \overline{b_{k}} e^{i k \xi}\right) e^{-i n \xi}\right\rangle_{L^{2}[0,2 \pi]} \\
& =\frac{1}{2 \pi}\left\langle\sum_{k \in \mathbb{Z}} a_{k} e^{-i k \xi}, \sum_{k \in \mathbb{Z}} \overline{b_{n-k}} e^{-i k \xi}\right\rangle_{L^{2}[0,2 \pi]}=\sum_{k \in \mathbb{Z}} a_{k} b_{n-k}
\end{aligned}
$$

by Parseval's identity. Hence the conclusion follows.

For any $\phi(t) \in L^{2}(\mathbb{R})$, let

$$
H_{\phi}(\xi):=\sum_{n \in \mathbb{Z}}|\hat{\phi}(\xi+2 n \pi)| \text { and } \quad C_{\phi}(t):=\sum_{n \in \mathbb{Z}}|\phi(t+n)|^{2} .
$$




\section{Main Result and an example}

In the following, let $\phi(t) \in L^{2}(\mathbb{R})$ be a scaling function of an MRA $\left\{V_{j}\right\}_{j \in \mathbb{Z}}$ and $\psi(t)$ the associated wavelet, of which we always assume that $H_{\phi}(\xi)$ and $H_{\psi}(\xi)$ are in $L^{\infty}[0,2 \pi]$. Then (cf. Proposition 2.4 in [8]) $\phi(t)$ and $\psi(t) \in L^{2}(\mathbb{R}) \cap$ $C(\mathbb{R})$ and $\sup C_{\phi}(t)<\infty, \sup C_{\psi}(t)<\infty$. Hence for any $\mathbf{c}=\left\{c_{n}\right\}_{n \in \mathbb{Z}} \in l^{2}$, $(\mathbf{c} * \phi)(t):=\sum_{n \in \mathbb{Z}} c_{n} \phi(t-n)$ converges both in $L^{2}(\mathbb{R})$ and uniformly in $\mathbb{R}$ so that each $V_{j} \subset L^{2}(\mathbb{R}) \cap C(\mathbb{R}), j \in \mathbb{Z}$.

Let $\mathcal{L}_{j}[\cdot]$ be the LTI (linear time invariant) systems with frequency responses $M_{j}(\xi) \in L^{2}(\mathbb{R}) \cap L^{\infty}(\mathbb{R})$ for $j=1,2$. Then

$$
\mathcal{L}_{j}[f](t)=\mathcal{F}^{-1}\left(\hat{f} M_{j}\right)(t) \in L^{2}(\mathbb{R}) \cap C(\mathbb{R}), f \in L^{2}(\mathbb{R}) \text { and } j=1,2
$$

and $\lim _{|t| \rightarrow \infty} \mathcal{L}_{j}[f](t)=0$ by the Riemann-Lebesgue Lemma since $\hat{f}(\xi) M_{j}(\xi) \in$ $L^{1}(\mathbb{R})$.

Moreover by the Poisson summation formula (cf. Lemma 5.1 in [8]), for any fixed $t \in \mathbb{R}$ and $j=1,2$,

$$
\sum_{n \in \mathbb{Z}} \mathcal{L}_{j}[\phi](t+n) e^{-i n \xi}=\sum_{n \in \mathbb{Z}} \hat{\phi}(\xi+2 n \pi) M_{j}(\xi+2 n \pi) e^{i t(\xi+2 n \pi)}
$$

and

$$
\sum_{n \in \mathbb{Z}} \mathcal{L}_{j}[\psi](t+n) e^{-i n \xi}=\sum_{n \in \mathbb{Z}} \hat{\psi}(\xi+2 n \pi) M_{j}(\xi+2 n \pi) e^{i t(\xi+2 n \pi)}
$$

are in $L^{\infty}[0,2 \pi]$ as functions in $\xi$ since $H_{\phi}(\xi), H_{\psi}(\xi) \in L^{\infty}[0,2 \pi]$.

In particular, $A_{i, j}(\xi)=A_{i, j}(\xi+2 \pi) \in L^{\infty}[0,2 \pi]$ for $i, j=1$, 2 , where

$$
A_{1, j}(\xi):=\sum_{n \in \mathbb{Z}} \mathcal{L}_{j}[\phi](n) e^{-i n \xi}, \quad A_{2, j}(\xi):=\sum_{n \in \mathbb{Z}} \mathcal{L}_{j}[\psi](n) e^{-i n \xi}
$$

Let

$$
A(\xi)=\left[A_{i, j}(\xi)\right]_{i, j=1}^{2} .
$$

Then for any $f(t)=\sum_{k \in \mathbb{Z}} c_{1, k} \phi(t-k)+\sum_{k \in \mathbb{Z}} c_{2, k} \psi(t-k) \in V_{1}$, where $\left\{c_{1, k}\right\}_{k \in \mathbb{Z}}$ and $\left\{c_{2, k}\right\}_{k \in \mathbb{Z}}$ in $l^{2}$, we have for $i=1,2$ and $n \in \mathbb{Z}$

$$
\mathcal{L}_{i}(f)(t)=\sum_{k \in \mathbb{Z}} c_{1, k} \mathcal{L}_{i}(\phi)(t-k)+\sum_{k \in \mathbb{Z}} c_{2, k} \mathcal{L}_{i}(\psi)(t-k),
$$

which converges both in $L^{2}(\mathbb{R})$ and absolutely on $\mathbb{R}$. In particular

$$
\mathcal{L}_{i}(f)(n)=\sum_{k \in \mathbb{Z}} c_{1, k} \mathcal{L}_{i}(\phi)(n-k)+\sum_{k \in \mathbb{Z}} c_{2, k} \mathcal{L}_{i}(\psi)(n-k), n \in \mathbb{Z} \text { and } i=1,2 .
$$


Lemma 3. Assume that $\operatorname{det} A(\xi) \neq 0$ a.e. in $[0,2 \pi]$. Let $\lambda_{1, B}(\xi) \leq \lambda_{2, B}(\xi)$ be eigenvalues of the Hermitian matrix $B(\xi)=\left(A(\xi)^{*} A(\xi)\right)^{-1}$. If $\|\operatorname{det} A(\xi)\|_{0}>$ 0 , then

$$
0<\left\|\lambda_{1, B}(\xi)\right\|_{0} \leq\left\|\lambda_{2, B}(\xi)\right\|_{\infty}<\infty .
$$

Proof. Since $B(\xi)$ is a nonsingular positive semi-definite Hermitian matrix a.e. in $[0,2 \pi]$,

$$
0<\lambda_{1, B}(\xi) \leq \lambda_{2, B}(\xi) \text { a.e. in }[0,2 \pi] .
$$

Since $A_{i, j}(\xi) \in L^{\infty}[0,2 \pi]$ and $\|\operatorname{det} A(\xi)\|_{0}>0$, all entries of $B(\xi)$ are also in $L^{\infty}[0,2 \pi]$ so that the characteristic equation of $B(\xi)$ is of the form

$$
\lambda(\xi)^{2}+f(\xi) \lambda(\xi)+g(\xi)=0
$$

where $f(\xi)$ and $g(\xi)$ are real-valued functions in $L^{\infty}[0,2 \pi]$. Hence $0<\left\|\lambda_{2, B}(\xi)\right\|_{\infty}<\infty$. Since $\lambda_{1, B}(\xi) \lambda_{2, B}(\xi)=\operatorname{det} B(\xi)=|\operatorname{det} A(\xi)|^{-2}$,

$$
\|\operatorname{det} A(\xi)\|_{\infty}^{-2} \leq \lambda_{1, B}(\xi) \lambda_{2, B}(\xi) \leq\|\operatorname{det} A(\xi)\|_{0}^{-2} \text { a.e. in }[0,2 \pi]
$$

so that $0<\|\operatorname{det} A(\xi)\|_{\infty}^{-2}\left\|\lambda_{2, B}(\xi)\right\|_{\infty}^{-1} \leq \lambda_{1, B}(\xi)$ a.e. in $[0,2 \pi]$.

Definition 2. For any $f_{1}(t)$ and $f_{2}(t)$ in $L^{2}(\mathbb{R})$, let $F(\xi):=\left[F_{i, j}(\xi)\right]_{i, j=1}^{2}$ be the Gramian of $\left\{f_{1}, f_{2}\right\}$, where $F_{i, j}(\xi):=\sum_{k \in \mathbb{Z}} \hat{f}_{i}(\xi+2 k \pi) \overline{\hat{f}_{j}(\xi+2 k \pi)}$.

Then as a Hermitian matrix, $F(\xi)$ has real eigenvalues.

Proposition 1. ([9]) Let $\lambda_{1, F}(\xi) \leq \lambda_{2, F}(\xi)$ be eigenvalues of the Gramian $F(\xi)$ of $\left\{f_{1}, f_{2}\right\}$. Then $\left\{f_{1}(t-n), f_{2}(t-n): n \in \mathbb{Z}\right\}$ is a Riesz sequence if and only if

$$
0<\left\|\lambda_{1, F}(\xi)\right\|_{0} \leq\left\|\lambda_{2, F}(\xi)\right\|_{\infty}<\infty .
$$

Lemma 4. Assume $\|\operatorname{det} A(\xi)\|_{0}>0$. Let $\left[\begin{array}{c}\hat{S}_{1}(\xi) \\ \hat{S}_{2}(\xi)\end{array}\right]:=A(\xi)^{-1}\left[\begin{array}{c}\hat{\phi}(\xi) \\ \hat{\psi}(\xi)\end{array}\right]$ and $S_{i}(t):=\mathcal{F}^{-1}\left(\hat{S}_{i}\right)(t)$ for $i=1,2$. Then $S_{i}(t) \in V_{1} \quad$ for $i=1,2$ and $\left\{S_{i}(t-n): i=1,2\right.$ and $\left.n \in \mathbb{Z}\right\}$ is a Riesz sequence.

Proof. Let $A(\xi)^{-1}=C(\xi)=\left[C_{i, j}(\xi)\right]_{i, j=1}^{2}$. Since $C_{i, j}(\xi) \in L^{\infty}(\mathbb{R}), \hat{S}_{i}(\xi)=$ $C_{i, 1}(\xi) \hat{\phi}(\xi)+C_{i, 2}(\xi) \hat{\psi}(\xi) \in L^{2}(\mathbb{R})$ for $i=1,2$. Since $C_{i, j}(\xi)=C_{i, j}(\xi+$ $2 \pi) \in L^{\infty}[0,2 \pi]$, we may expand $C_{i, j}(\xi)$ into its Fourier series $C_{i, j}(\xi)=$ $\sum_{k \in \mathbb{Z}} c_{i, j, k} e^{-i k \xi}$ where $\left\{c_{i, j, k}\right\}_{k \in \mathbb{Z}} \in l^{2}$. Then by Lemma 1 ,

$$
\hat{S}_{i}(\xi)=\sum_{k \in \mathbb{Z}}\left(c_{i, 1, k} e^{-i k \xi} \hat{\phi}(\xi)+c_{i, 2, k} e^{-i k \xi} \hat{\psi}(\xi)\right)
$$


so that

$$
S_{i}(t):=\mathcal{F}^{-1}\left(\hat{S}_{i}\right)(t)=\sum_{k \in \mathbb{Z}}\left(c_{i, 1, k} \phi(t-k)+c_{i, 2, k} \psi(t-k)\right) \in V_{1} .
$$

Let $G(\xi)$ and $S(\xi)$ be the Gramians of $\{\phi, \psi\}$ and $\left\{S_{1}, S_{2}\right\}$ respectively and $\lambda_{1, G}(\xi) \leq \lambda_{2, G}(\xi)$ and $\lambda_{1, S}(\xi) \leq \lambda_{2, S}(\xi)$ the eigenvalues of $G(\xi)$ and $S(\xi)$ respectively. Then $S(\xi)=C(\xi) G(\xi) C(\xi)^{*}$. Let $U_{S}(\xi)$ and $U_{G}(\xi)$ be unitary matrices, which diagonalize $S(\xi)$ and $G(\xi)$ respectively, i.e.,

$$
S(\xi)=U_{S}(\xi)\left[\begin{array}{cc}
\lambda_{1, S}(\xi) & 0 \\
0 & \lambda_{2, S}(\xi)
\end{array}\right] U_{S}(\xi)^{*}
$$

and

$$
G(\xi)=U_{G}(\xi)\left[\begin{array}{cc}
\lambda_{1, G}(\xi) & 0 \\
0 & \lambda_{2, G}(\xi)
\end{array}\right] U_{G}(\xi)^{*}
$$

Then

$$
\left[\begin{array}{cc}
\lambda_{1, S}(\xi) & 0 \\
0 & \lambda_{2, S}(\xi)
\end{array}\right]=R(\xi)\left[\begin{array}{cc}
\lambda_{1, G}(\xi) & 0 \\
0 & \lambda_{2, G}(\xi)
\end{array}\right] R(\xi)^{*}
$$

where

$$
R(\xi)=U_{S}(\xi)^{*} C(\xi) U_{G}(\xi):=\left[\begin{array}{ll}
R_{1,1}(\xi) & R_{1,2}(\xi) \\
R_{2,1}(\xi) & R_{2,2}(\xi)
\end{array}\right]
$$

so that

$$
\begin{aligned}
& \lambda_{1, S}(\xi)=\lambda_{1, G}(\xi)\left|R_{1,1}(\xi)\right|^{2}+\lambda_{2, G}(\xi)\left|R_{1,2}(\xi)\right|^{2} ; \\
& \lambda_{2, S}(\xi)=\lambda_{1, G}(\xi)\left|R_{2,1}(\xi)\right|^{2}+\lambda_{2, G}(\xi)\left|R_{2,2}(\xi)\right|^{2} .
\end{aligned}
$$

On the other hand,

$$
\begin{aligned}
R(\xi) R(\xi)^{*} & =U_{S}(\xi)^{*} C(\xi) C(\xi)^{*} U_{S}(\xi)=U_{S}(\xi)^{*} B(\xi) U_{S}(\xi) \\
& =U_{S}(\xi)^{*} U_{B}(\xi)\left[\begin{array}{cc}
\lambda_{1, B}(\xi) & 0 \\
0 & \lambda_{2, B}(\xi)
\end{array}\right] U_{B}(\xi)^{*} U_{S}(\xi),
\end{aligned}
$$

where $U_{B}(\xi)$ is the unitary matrix such that

$$
B(\xi)=U_{B}(\xi)\left[\begin{array}{cc}
\lambda_{1, B}(\xi) & 0 \\
0 & \lambda_{2, B}(\xi)
\end{array}\right] U_{B}(\xi)^{*}
$$

with $\lambda_{1, B}(\xi) \leq \lambda_{2, B}(\xi)$. Set $U_{S}(\xi)^{*} U_{B}(\xi)=\left[D_{i, j}(\xi)\right]_{i, j=1}^{2}$, which is also a unitary matrix. Then we have from diagonal entries of both sides of (4),

$$
\begin{aligned}
& \left|R_{1,1}(\xi)\right|^{2}+\left|R_{1,2}(\xi)\right|^{2}=\lambda_{1, B}(\xi)\left|D_{1,1}(\xi)\right|^{2}+\lambda_{2, B}(\xi)\left|D_{1,2}(\xi)\right|^{2} ; \\
& \left|R_{2,1}(\xi)\right|^{2}+\left|R_{2,2}(\xi)\right|^{2}=\lambda_{1, B}(\xi)\left|D_{2,1}(\xi)\right|^{2}+\lambda_{2, B}(\xi)\left|D_{2,2}(\xi)\right|^{2} .
\end{aligned}
$$


Then we have a.e. in $[0,2 \pi]$ (from $(2),(3),(5)$, and (6))

$$
\begin{aligned}
& \lambda_{1, S}(\xi) \geq \lambda_{1, G}(\xi)\left(\left|R_{1,1}(\xi)\right|^{2}+\left|R_{1,2}(\xi)\right|^{2}\right) \geq \lambda_{1, G}(\xi) \lambda_{1, B}(\xi) ; \\
& \lambda_{2, S}(\xi) \leq \lambda_{2, G}(\xi)\left(\left|R_{, 21}(\xi)\right|^{2}+\left|R_{2,2}(\xi)\right|^{2}\right) \leq \lambda_{2, G}(\xi) \lambda_{2, B}(\xi)
\end{aligned}
$$

since $\left|D_{1,1}(\xi)\right|^{2}+\left|D_{1,2}(\xi)\right|^{2}=\left|D_{2,1}(\xi)\right|^{2}+\left|D_{2,2}(\xi)\right|^{2}=1$ a.e. in $[0,2 \pi]$. Hence

$$
\begin{gathered}
0<\left\|\lambda_{1, G}(\xi)\right\|_{0}\left\|\lambda_{1, B}(\xi)\right\|_{0} \leq\left\|\lambda_{1, S}(\xi)\right\|_{0} \\
\leq\left\|\lambda_{2, S}(\xi)\right\|_{\infty} \leq\left\|\lambda_{2, G}(\xi)\right\|_{\infty}\left\|\lambda_{2, B}(\xi)\right\|_{\infty}<\infty
\end{gathered}
$$

by Lemma 3 and Proposition 4 so that $\left\{S_{i}(t-n): i=1,2\right.$ and $\left.n \in \mathbb{Z}\right\}$ is a Riesz sequence by Proposition 4 .

Now we are ready to give the main result of this work.

Theorem 1. There exist $S_{i}(t) \in V_{1}(i=1,2)$ such that $\left\{S_{i}(t-n): i=\right.$ $1,2$ and $n \in \mathbb{Z}\}$ is a Riesz basis of $V_{1}$ for which two-channel sampling formula

$$
f(t)=\sum_{n \in \mathbb{Z}} \mathcal{L}_{1}(f)(n) S_{1}(t-n)+\sum_{n \in \mathbb{Z}} \mathcal{L}_{2}(f)(n) S_{2}(t-n)
$$

holds for $f \in V_{1}$ if and only if $\|\operatorname{det} A(\xi)\|_{0}>0$. In this case

$$
S_{i}(t)=\mathcal{F}^{-1}\left(C_{i, 1}(\xi) \hat{\phi}(\xi)+C_{i, 2}(\xi) \hat{\psi}(\xi)\right)(t) \quad \text { for } i=1,2 .
$$

Proof. Assume $\|\operatorname{det} A(\xi)\|_{0}>0$ and define $S_{i}(t)$ by (8). Then $S_{i}(t) \in V_{1}(i=$ $1,2)$ and $\left\{S_{i}(t-n): i=1,2\right.$ and $\left.n \in \mathbb{Z}\right\}$ is a Riesz sequence by Lemma 5 . For any $f(t) \in V_{1}$

$$
f(t)=\sum_{k \in \mathbb{Z}} c_{1, k} \phi(t-k)+\sum_{k \in \mathbb{Z}} c_{2, k} \psi(t-k)
$$

where $\left\{c_{i, k}\right\}_{k \in \mathbb{Z}} \in l^{2}$ for $i=1,2$, we have by Lemma 1 ,

$$
\hat{f}(\xi)=\left(\sum_{k \in \mathbb{Z}} c_{1, k} e^{-i k \xi}\right) \hat{\phi}(\xi)+\left(\sum_{k \in \mathbb{Z}} c_{2, k} e^{-i k \xi}\right) \hat{\psi}(\xi) .
$$

Since $\left[\begin{array}{c}\hat{\phi}(\xi) \\ \hat{\psi}(\xi)\end{array}\right]=A(\xi)\left[\begin{array}{c}\hat{S}_{1}(\xi) \\ \hat{S}_{2}(\xi)\end{array}\right]$, we have by (1), (9) and Lemma 1

$$
\begin{aligned}
\hat{f}(\xi) & =\sum_{j=1}^{2}\left[\left(\sum_{k \in \mathbb{Z}} c_{1, k} e^{-i k \xi}\right) A_{1, j}(\xi)+\left(\sum_{k \in \mathbb{Z}} c_{2, k} e^{-i k \xi}\right) A_{2, j}(\xi)\right] \hat{S}_{j}(\xi)(10) \\
& =\sum_{n \in \mathbb{Z}}\left[\mathcal{L}_{1}[f](n) e^{-i n \xi} \hat{S}_{1}(\xi)\right]+\sum_{n \in \mathbb{Z}}\left[\mathcal{L}_{2}[f](n) e^{-i n \xi} \hat{S}_{2}(\xi)\right] .
\end{aligned}
$$


Taking the inverse Fourier transform on (10) gives (7), which implies $V_{1}=$ $\overline{\operatorname{span}}\left\{S_{i}(t-n): i=1,2\right.$ and $\left.n \in \mathbb{Z}\right\}$ so that $\left\{S_{i}(t-n): i=1,2\right.$ and $\left.n \in \mathbb{Z}\right\}$ is a Riesz basis of $V_{1}$. Conversely assume that there exist $S_{i}(t) \in V_{1}(i=1,2)$ such that $\left\{S_{i}(t-n): i=1,2\right.$ and $\left.n \in \mathbb{Z}\right\}$ is a Riesz basis of $V_{1}$ and (7) holds. In particular,

$$
\begin{aligned}
\phi(t) & =\sum_{n \in \mathbb{Z}} \mathcal{L}_{1}[\phi](n) S_{1}(t-n)+\sum_{n \in \mathbb{Z}} \mathcal{L}_{2}[\phi](n) S_{2}(t-n) ; \\
\psi(t) & =\sum_{n \in \mathbb{Z}} \mathcal{L}_{1}[\psi](n) S_{1}(t-n)+\sum_{n \in \mathbb{Z}} \mathcal{L}_{2}[\psi](n) S_{2}(t-n) .
\end{aligned}
$$

By taking Fourier transform and using Lemma 1, we have

$$
\left[\begin{array}{l}
\hat{\phi}(\xi) \\
\hat{\psi}(\xi)
\end{array}\right]=A(\xi)\left[\begin{array}{c}
\hat{S}_{1}(\xi) \\
\hat{S}_{2}(\xi)
\end{array}\right]
$$

We then have as in the proof of Lemma $5, G(\xi)=A(\xi) S(\xi) A(\xi)^{*}$, where $G(\xi)$ and $S(\xi)$ are Gramians of $\{\phi, \psi\}$ and $\left\{S_{1}, S_{2}\right\}$ respectively. Hence $\operatorname{det} G(\xi)=$ $\operatorname{det} S(\xi)|\operatorname{det} A(\xi)|^{2}$ so that

$$
|\operatorname{det} A(\xi)|^{2}=\frac{\operatorname{det} G(\xi)}{\operatorname{det} S(\xi)}=\frac{\lambda_{1, G}(\xi) \lambda_{2, G}(\xi)}{\lambda_{1, S}(\xi) \lambda_{2, S}(\xi)} \geq \frac{\lambda_{1, G}(\xi)^{2}}{\lambda_{2, S}(\xi)^{2}} \quad \text { a.e. in }[0,2 \pi] \text {, }
$$

where $\lambda_{1, G}(\xi) \leq \lambda_{2, G}(\xi)$ and $\lambda_{1, S}(\xi) \leq \lambda_{2, S}(\xi)$ are eigenvalues of $G(\xi)$ and $S(\xi)$ respectively. Therefore,

$$
|\operatorname{det} A(\xi)| \geq \frac{\lambda_{1, G}(\xi)}{\lambda_{2, S}(\xi)} \geq \frac{\left\|\lambda_{1, G}(\xi)\right\|_{0}}{\left\|\lambda_{2, S}(\xi)\right\|_{\infty}} \text { a.e. in }[0,2 \pi]
$$

so that $\|\operatorname{det} A(\xi)\|_{0}>0$ since both $\{\phi(t-n), \psi(t-n): n \in \mathbb{Z}\}$ and $\left\{S_{i}(t-\right.$ $n): i=1,2$ and $n \in \mathbb{Z}\}$ are Riesz sequences. Finally (8) comes from (11) immediately.

Note that if $\left\{\mathcal{L}_{i}(\phi)(n)\right\}_{n \in \mathbb{Z}}$ and $\left\{\mathcal{L}_{i}(\psi)(n)\right\}_{n \in \mathbb{Z}} \in l^{1}$, then $A_{i, j}(\xi) \in C[0,2 \pi]$ for $i, j=1,2$ so that $A_{i, j}(\xi) \in L^{\infty}[0,2 \pi]$ and $\|\operatorname{det} A(\xi)\|_{0}>0$ is equivalent to $\operatorname{det} A(\xi) \neq 0$ on $[0,2 \pi]$.

Example. (2-channel sampling in Paley-Wiener space)

Let $\phi(t)=$ sinct so that $V_{0}=\overline{\operatorname{span}}\{\phi(t-n): n \in \mathbb{Z}\}=P W_{\pi}$ and $V_{1}=$ $\overline{\operatorname{span}}\{\phi(2 t-n): n \in \mathbb{Z}\}=P W_{2 \pi}$. Then $V_{1}=V_{0} \oplus W_{0}$ where $W_{0}=\overline{\operatorname{span}}\{\psi(t-$ $n): n \in \mathbb{Z}\}$ and $\psi(t)=\left(\cos \frac{3}{2} \pi t\right)\left(\operatorname{sinc} \frac{1}{2} t\right)$. 
Note that $\hat{\phi}(\xi)=\frac{1}{\sqrt{2 \pi}} \chi_{[-\pi, \pi]}(\xi)$ and $\hat{\psi}(\xi)=\frac{1}{\sqrt{2 \pi}} \chi_{[-2 \pi,-\pi] \cup[\pi, 2 \pi]}(\xi)$, where $\chi_{E}(\cdot)$ is the characteristic function of a set $E$ in $\mathbb{R}$. We have by (12)

$$
\sum_{n \in \mathbb{Z}} \mathcal{L}_{i}[\phi](n) e^{-i n \xi}= \begin{cases}M_{i}(\xi) & , \xi \in[0, \pi) \\ M_{i}(\xi-2 \pi) & , \xi \in[\pi, 2 \pi]\end{cases}
$$

and

$$
\sum_{n \in \mathbb{Z}} \mathcal{L}_{i}[\psi](n) e^{-i n \xi}= \begin{cases}M_{i}(\xi-2 \pi) & , \xi \in[0, \pi) \\ M_{i}(\xi) & , \xi \in[\pi, 2 \pi]\end{cases}
$$

Hence

$$
A(\xi)=\left\{\begin{array}{cc}
{\left[\begin{array}{cc}
M_{1}(\xi) & M_{2}(\xi) \\
M_{1}(\xi-2 \pi) & M_{2}(\xi-2 \pi)
\end{array}\right]} & \text { on }[0, \pi) \\
{\left[\begin{array}{cc}
M_{1}(\xi-2 \pi) & M_{2}(\xi-2 \pi) \\
M_{1}(\xi) & M_{2}(\xi)
\end{array}\right]} & \text { on }[\pi, 2 \pi]
\end{array}\right.
$$

so that the determinant condition $\|\operatorname{det} A(\xi)\|_{0}>0$ is equivalent to $\|\operatorname{det} M(\xi)\|_{0}>0$ where

$$
M(\xi)=\left[\begin{array}{ll}
M_{1}(\xi) & M_{1}(\xi-2 \pi) \\
M_{2}(\xi) & M_{2}(\xi-2 \pi)
\end{array}\right]
$$

Take $M_{\tilde{1}}(\xi)=1$ and $M_{2}(\xi)=-i \operatorname{sgn} \xi$ so that $\mathcal{L}_{1}[f](t)=f(t)$ and $\mathcal{L}_{2}[f](t)=\tilde{f}(t)$ where $\tilde{f}(t)$ is the Hilbert transform of $f(t)$. Then

$$
M(\xi)=\left[\begin{array}{cc}
1 & 1 \\
-i & i
\end{array}\right]
$$

so that $\|\operatorname{det} M(\xi)\|_{0}=2$. As a consequence, the sampling formula holds on $V_{1}=P W_{2 \pi}$. In fact, we have from (11),

$$
\left[\begin{array}{c}
\hat{S}_{1}(\xi) \\
\hat{S}_{2}(\xi)
\end{array}\right]= \begin{cases}\frac{1}{2 \sqrt{2 \pi}}\left[\begin{array}{c}
1 \\
-i
\end{array}\right] & \text { on }[-2 \pi, 0) \\
\frac{1}{2 \sqrt{2 \pi}}\left[\begin{array}{l}
1 \\
i
\end{array}\right] & \text { on }[0,2 \pi] \\
{\left[\begin{array}{l}
0 \\
0
\end{array}\right]} & \text { otherwise }\end{cases}
$$

so that

$$
f(t)=\sum_{n \in \mathbb{Z}} f(n) \operatorname{sinc} 2(t-n)-\sum_{n \in \mathbb{Z}} \tilde{f}(n) \sin \pi(t-n) \operatorname{sinc}(t-n), f \in P W_{2 \pi} .
$$


As another example, take $M_{1}(\xi)=1$ and $M_{2}(\xi)=i \xi$ so that $\mathcal{L}_{1}[f](t)=f(t)$ and $\mathcal{L}_{2}[f](t)=f^{\prime}(t)$. Then

$$
M(\xi)=\left[\begin{array}{cc}
1 & 1 \\
i \xi & i(\xi-2 \pi)
\end{array}\right]
$$

so that $\|\operatorname{det} M(\xi)\|_{0}=2 \pi$. By the similar procedure as above, we obtain a sampling formula

$f(t)=\sum_{n \in \mathbb{Z}} f(n) \operatorname{sinc}^{2} 2(t-n)+\frac{1}{\pi} \sum_{n \in \mathbb{Z}} f^{\prime}(n) \sin \pi(t-n) \operatorname{sinc}(t-n), f \in P W_{2 \pi}$.

Acknowledgments : This work is partially supported by the National Research Foundation of Korea (NRF) (2012R1A1A2038650)

\section{References}

[1] A. Aldroubi and K. Gröchenig, Nonuniform sampling and reconstruction in shift-invariant spaces, SIAM Review, vol. 43, no. 4, pp. 585-620, 2001.

[2] W. Chen and S. Itoh, A sampling theorem for shift-invariant subspace, IEEE Trans. Signal Processing, vol. 46, pp. 2822-2824, 1998.

[3] O. Christensen, An Introduction to frames and Riesz Bases, Birkhäuser, 2002 .

[4] J. R. Higgins, Sampling theory in Fourier and signal analysis, Oxford University Press, 1996.

[5] Y. M. Hong, J. M. Kim, K. H. Kwon and E. H. Lee, Channeled sampling in shift invariant spaces, Int. J. Wavelets Multiresolut. Inf. Process., vol. 5, pp. 753-767, 2007.

[6] A. J. E. M. Janssen, The Zak-transform and sampling theorem for wavelet subspaces, IEEE Trans. Signal Processing, vol. 41, pp. 3360-3364, 1993.

[7] A. J. Jerri, The Shannon sampling theorem-Its various extentions and applications: A tutorial review, Proc. IEEE, vol. 65, pp. 1565-1596, 1977.

[8] J. M. Kim and K. H. Kwon, Sampling expansion in shift invariant spaces, Int. J. Wavelets Multiresolut. Inf. Process., vol. 6, no. 2, pp. 223-248, 2008. 
[9] A. Ron and Z. Shen, Frames and stable bases for shift-invariant subspaces of $L^{2}(\mathbb{R})$, Canadian J. Math., vol. 47, pp.1051-1094, 1995.

[10] M. Unser, Sampling-50 years after Shannon, Proc. IEEE, vol. 88, pp. 567-587, 2000.

[11] G. G. Walter, A sampling theorem for wavelet subspaces, IEEE Trans. Inform. Theory, vol. 38, pp. 881-884, 1992.

[12] P. Zhao, G. Liu and S. Itoh, Frames and sampling theorem for trnaslation-invariant subspaces, IEEE Signal Processing Letters, vol. 11, pp. 8-11, 2004.

J.M. KIM,

Faculty of Mathematics and Computer Sciences,

Korea Science Academy of KAIST,

Korea

e-mail: frankim@gmail.com

K. H. KWON,

Faculty of Mathematical Sciences,

Korea Advanced Institute of Science and Technology

Korea

e-mail: khkwon@kaist.edu 
